\title{
Production and perception asymmetries in the Canadian vowel shift
}

Thomas Kettig ${ }^{1}$, Bodo Winter ${ }^{2}$

${ }^{1}$ Theoretical and Applied Linguistics, University of Cambridge

${ }^{2}$ Cognitive and Information Sciences, University of California, Merced

https://doi.org/10.36505/ExLing-2015/06/0008/000245

\begin{abstract}
The Canadian Shift (CS), a lowering and backing of the KIT, DRESS, and TRAP vowels, has been extensively investigated in the speech of English Canadians. However, its effect on the perceptual categorization of vowels has received little attention. The role of perception in ongoing vowel shifts remains comparatively under-researched. By testing participants both in production and in perception, this study gives a unique view into an ongoing sound change. Participants from Montreal in two age groups were recorded reading a list of 44 sentences containing words with stressed $/ \varepsilon /, / \mathfrak{x} /, / \mathrm{\jmath} /$, and $/ \Lambda /$ vowels. Participants also categorized 96 synthesized vowel stimuli. While the production data clearly replicated the CS, in perception, shift-leaders did not categorize vowels very differently.
\end{abstract}

Key words: Canadian Vowel Shift; English; sound change; speech perception

\section{Introduction}

The Canadian Shift (CS) describes a systematic lowering and backing of the KIT, DRESS, and TRAP vowels. However, Boberg (2008: 137) concludes in an overview of the Canadian English vowel system that "the regional profile of the Canadian Shift is far from clear." This paper follows up on earlier work from Boberg (2005) and addresses the apparent-time trajectory of the CS in a community of English speakers in Montreal. We do not only investigate the ongoing sound in speech production, but also in speech perception (cf. Kendall \& Fridland 2012; Thomas 2002), allowing us to see the extent to which speakers who are leading the change in production are also leading the change in perception.

\section{Methodology}

To control for the effect of local ethnolect, this study is limited to the Jewish community of Montreal (pop. 80,000). Our group contained 12 young speakers (born 1984 or later, 5 female, 7 male) and 16 older speakers (born 1961 or earlier, 5 female, 11 male).

Each participant performed two experiments. In Experiment I, participants spoke a list of 44 sentences containing words with stressed $/ \varepsilon /, / \mathfrak{x} /, / \mathrm{\jmath} /$, and $/ \Lambda /$ vowels. The target words were controlled for voicing, place of articulation, and manner of articulation of the consonant following the vowel. In

ExLing 2015: Proceedings of 6th Tutorial and Research Workshop on Experimental Linguistics, 26-27 June 2015, Athens, Greece 
Experiment II, we presented human-sounding synthetic vowels to participants, each $250 \mathrm{~ms}$ in duration. In each trial, four large buttons of equal size, with the labels BAT, BET, BUT, and BOUGHT, appeared on a screen for participants to select as they listened to 96 stimuli, which formed an F1 continuum from 700 $\mathrm{Hz}$ to $950 \mathrm{~Hz}$ and a F2 continuum from $1200 \mathrm{~Hz}$ to $1950 \mathrm{~Hz}$. Stimuli were played in the same, random order for every participant.

\section{Results}

Figure 1 displays the Lobanov-normalized vowel space, with each vowel symbol representing the mean F1/F2 of one speaker, plus confidence ellipses. Age differences are most pronounced for $/ \mathfrak{x} /$ and $/ \varepsilon /$. Both are retracted for younger speakers. The orientation of the ellipses shows that the change of $/ \mathfrak{x} /$ is primarily one in F2. On the other hand, the change of $/ \varepsilon /$ is expressed both in F1 and F2, although it is more pronounced for F2. Linear mixed effects models confirm these observations: There are significant age differences in F2 for $/ \mathfrak{x} /(\mathrm{p}<0.001)$ and $/ \varepsilon /(\mathrm{p}=0.03)$, but not for $/ \Lambda /$ and $/ \mathrm{\rho} /(\mathrm{p}>0.1)$. There was also a nearly significant effect in F1 for $/ \varepsilon /(\mathrm{p}=0.06)$.
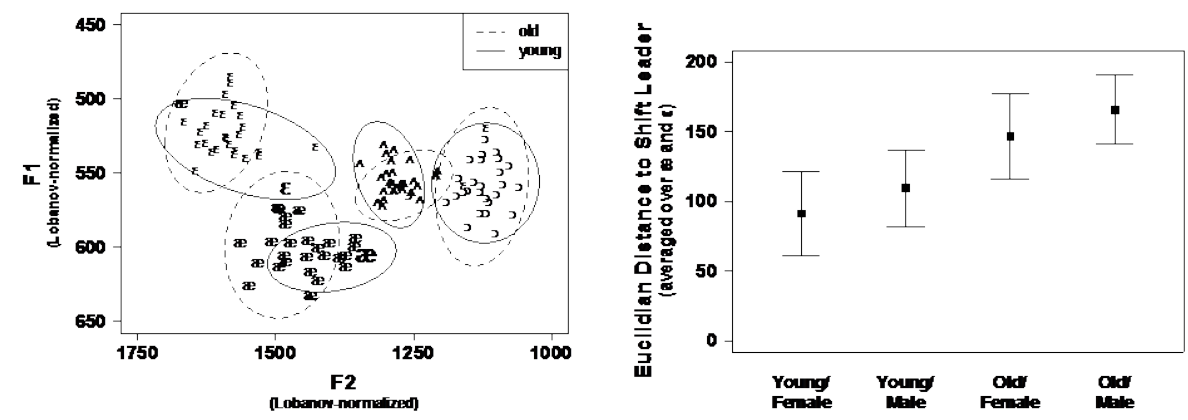

Figure 1. Means for each speaker's Figure 2. Linear mixed effects model vowels (production), plus 95\% predictions for Euclidian distance to confidence ellipses (solid line for shift leader (error bars indicate 95\% younger speakers, dotted line for older confidence interval). speakers).

Based on Figure 1, we selected a speaker whose $/ \mathfrak{x} /$ mean was most retracted, as well as a speaker whose $/ \varepsilon /$ mean was most lowered. Since these two speakers have the most extreme values in the direction of the shift, they can be thought of as "shift leaders," highlighted in boldface in Figure 1. We used the two speakers' mean F1/F2 values of $/ \mathfrak{x} /$ and $/ \varepsilon /$ to calculate Euclidian distances for each vowel. This Euclidian distance can be interpreted as indicating a measure of "shift leadingness" for every vowel. Figure 2 shows this measure for the age/gender groups in our data. A mixed model analysis 
reveals that the effect of "Group" is significant $(p<0.001)$, with young women leading the change. Older men are the least advanced, with younger men and older women falling in between these two extremes.

In Experiment II, we investigated how age differences affect the perception of $/ \mathfrak{x}, \varepsilon, \Lambda, \rho /$. Figure 3 shows the average $\mathrm{F} 1 / \mathrm{F} 2$ values along the synthetic continuum for which each speaker responded with a specific vowel. As expected, values with low F1 and high F2 are categorized as $/ \varepsilon /$, values with high F1 and mid to high F2 as /æ/, and so on. The confidence ellipses indicate nearly complete overlap between young and older speakers in classifying tokens as $/ \mathfrak{x} /$. On the other hand, some of the younger listeners categorize tokens with relatively low $\mathrm{F} 2$ as $/ \varepsilon /$. For $/ \Lambda /$ and $/ \rho /$, the categorization results are not straightforward. There is considerable overlap between the F1/F2 distributions of these two vowels, and there are noticeable age differences. A generalized additive model with F1 and F2 as tensor product splines was fit for each vowel's categorization (vowel vs. rest), modeling how categorization behaviour changed as a function of vowel acoustics as well as age. Interestingly, the model indicates no significant interaction between F1/F2 and age for / $/$ $(p=0.26)$, nor does it indicate an age interaction for $/ \varepsilon /(p=0.13)$. However, there are significant age interaction effects for $/ \Lambda /(p<0.001)$ and $/ \mathrm{\rho} /(\mathrm{p}=0.04)$.

This finding indicates a notable discrepancy between speech production and speech perception in the CS: significant age differences exist for $/ \mathfrak{x} /$ and $/ \varepsilon /$ in production, but not for $/ \Lambda /$ and $/ \mathrm{\rho} /$. The reverse is true in perception, with $/ \Lambda /$ and $/ \mathrm{o} /$ showing bigger perceptual differences than $/ \mathfrak{x} /$ and $/ \varepsilon /$.
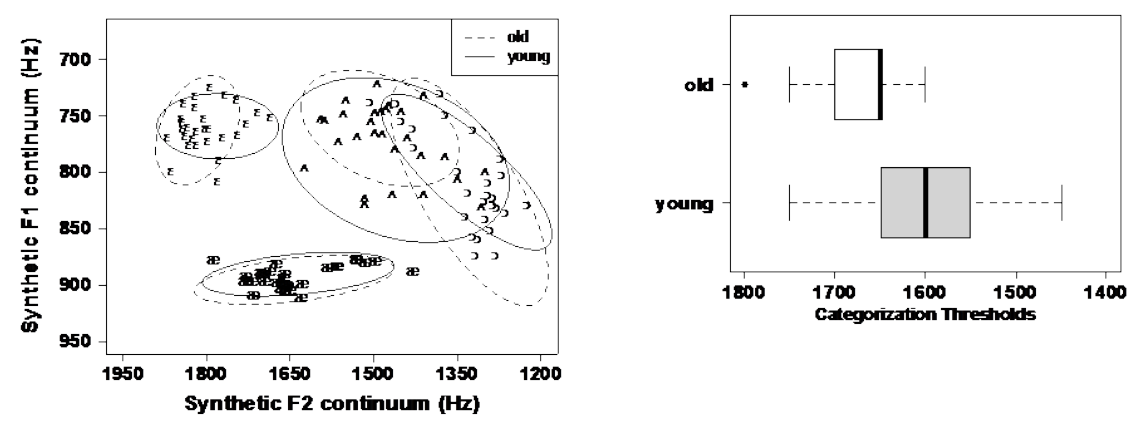

Figure 3. Means for each listener's Figure 4. Categorization boundary vowels (perception), plus confidence between $/ \mathfrak{x} /$ and $/ \varepsilon /$ based on age intervals (solid line for younger, dotted group and F2. for older).

However, if we specifically focus on $/ \mathfrak{x} /$ versus $/ \varepsilon /$ categorizations (ignoring all other responses), there are significant age differences. Figure 4 shows a measure of $/ \mathfrak{x} \sim \mathcal{E} /$ categorization behaviour, generated by performing 
binary partitioning on each participant's $/ \mathfrak{\varepsilon} \sim \mathcal{\varepsilon} /$ F2 categorization curves. As can be seen, this measure correlates with age, and there is a significant effect of age (permutation test, $\mathrm{p}=0.036$ ). Younger speakers accept more retracted vowels (lower F2) as realizations of $/ \mathfrak{x} /$. In other words, the point at which they switch to $/ \varepsilon /$ occurs significantly later on the F2 continuum.

\section{Discussion}

Production results demonstrate that $/ \mathfrak{x} /$ and $/ \varepsilon /$ are shifting in apparent time in the vowel spaces of English-speaking Montrealers (Figure 1). An analysis of inter-speaker variation shows a significant degree of ordered heterogeneity, with young women leading the change and older males retaining the most conservative pronunciations, the typical progression for a sound change advancing below the level of consciousness. Though Boberg's (2005) study of Montreal found $/ \varepsilon /$ to be retracting and $/ \mathfrak{x} /$ to be both lowering and retracting, it seems as though the operation of the CS in Montreal now involves the retraction of $/ \mathfrak{x} /$ without any accompanying lowering, while $/ \varepsilon /$ is simultaneously backed and somewhat lowered. One implication of this finding could be that over the last decade, / $\mathfrak{x} /$ could have lowered as far in the vowel space as it will go, and is now only retracting (cf. Roeder \& Jarmasz 2010).

Although the categorization behaviour between $/ \mathfrak{x} /$ and $/ \varepsilon /$ is significantly affected by age (but only for F2), there are overall less pronounced differences in perception than in production. In fact, those vowels that do not show age differences in production, $/ \Lambda /$ and $/ \supset /$, show the biggest age differences in perception. This finding suggests that in an ongoing change, speakers' productions may differ, but their perception needs to accommodate the fact that both new and old variants need to be heard: younger speakers need to understand older speakers who do not participate as strongly in the shift. Thus, in the Canadian Vowel Shift, both production and perception are changing, but production changes more than perception.

\section{References}

Boberg, C. 2005. The Canadian Shift in Montreal. Language Variation and Change 17: 133154.

Boberg, C. 2008. Regional Phonetic Differentiation in Standard Canadian English. Journal of English Linguistics 36: 129.

Kendall, T. \& Fridland, V. 2012. Variation in perception and production of mid front vowels in the US Southern Vowel Shift. Journal of Phonetics 40: 289-306.

Roeder, R. \& L.-G. Jarmasz. 2010. The Canadian Shift in Toronto. The Canadian Journal of Linguistics 55(3): 387-404.

Thomas, E. R. 2002. Sociophonetic Applications of Speech Perception Experiments. American Speech 77(2): 115-147. 